\title{
The Role of Surgical Treatment in Traumatic Subdural Hygroma: A Pilot Study
}

\author{
Shahrokh Yousefzadeh-Chabok ${ }^{1}$, Marieh Hosseinpour ${ }^{2}$, Zahra Mohtasham-Amiri ${ }^{3}$, Ehsan Kazemnejad- \\ Leili ${ }^{4}$, Babak Alijani ${ }^{\text {* }}$ \\ ${ }^{1}$ MD, Professor of Neurosurgery, Neurosurgery Department, Poursina Hospital, Guilan University of Medical Sciences, Rasht, Guilan, Iran \\ ${ }^{2}$ MSc in Physiology, Guilan Road Trauma Research Center, Guilan University of Medical Sciences, Rasht, Guilan, Iran \\ ${ }^{3}$ MD, Professor of Social Medicine, Guilan Road Trauma Research Center, Guilan University of Medical Sciences, Rasht, Guilan, Iran \\ ${ }^{4}$ Ph.D, Associate Professor of Biostatistics, Guilan University of Medical Sciences, Rasht, Guilan, Iran \\ ${ }^{5}$ M.D, Assistant Professor of Neurosurgery, Guilan Road Trauma Research Center, Department of Neurosurgery, Poursina Hospital, Guilan \\ University of Medical Sciences, Rasht, Guilan, Iran \\ * Corresponding Author: Poursina Hospital, Guilan University of Medical Sciences, Rasht, Guilan, Iran. Tel: +981333338373, Fax: +981333338373. \\ E-mail: drbabakalij@gmail.com
}

Article Type: Research Article

Received: October 27, 2014, Last revised: February 27, 2015, Accepted: September 22, 2015

\section{Abstract}

Background and Aim: Traumatic subdural hygroma is the accumulation of CSF (cerebrospinal fluid) in subdural space following head trauma. The mass effect of hygroma on brain can impinge on consciousness. There are still many ambiguities on indications of hygroma surgery. This is an 11-year follow-up study which involves the patients suffering traumatic subdural hygroma who underwent surgery.

Methods \& Materials/Patients: In this retrospective study, clinical records of 16 patients who were operated due to traumatic subdural hygroma were studied. The data from existing records in the hospital were collected and analyzed. They were then analyzed by Repeated Measures ANOVA using SPSS (Version 18). The differences were considered statistically significant at $\mathrm{P} \leq 0.05$.

Results: In this study, there were 13 men $(81.3 \%)$ and 3 women $(18.7 \%$ ) (Mean age $=62$ years old). In $87.75 \%$ of patients, hygroma was diagnosed 6 days after head injury. It wasunilateral in majority of patients $(56.3 \%)$ and located in fronto-parietal area $(81.3 \%)$. The most frequent concomitant injuries were contusions $(25 \%)$ and subarachnoid hemorrhage (18.8\%), respectively. GCS trend on admission and at discharge was significantly different fromthat of hygroma formation $(\mathrm{P}<0.05)$. One-fourth of patients had recurrence of hygroma after surgery. All patients (except one) had good outcome.

Conclusion: Subdural hygroma is a delayed lesion and surgical treatment improves the level of consciousness (LOC) in afflicted patients.

Keywords: Subdural Hygroma; Head injury; Surgery

Please cite this paper as: Yousefzadeh-Chabok Sh, Hosseinpour M, Mohtasham-Amiri Z, Kazemnejad-Leili, E, Alijani B. The Role of Surgical Treatment in Traumatic Subdural Hygroma: A Pilot Study. Iran. J. Neurosurg. 2015;1(2):40-43.

\section{Introduction}

Traumatic brain injury (TBI) still remains an important concern and one of the leading causes of morbidity and mortality (1). Cerebral injuries such as subarachnoid hemorrhage (SAH), epidural hematoma (EDH) and subdural hematoma $(\mathrm{SDH})$ have been already described but subdural hygroma (SDG) still remains vague (2). SDG is the leakage and accumulation of cerebrospinal fluid (CSF), usually with modified composition in subdural space $(2,3)$. Generally subdural space does not exist (normally) but a mild trauma can separate the space between dura matter and arachnoid (3) which includes "an interface layer composed of an arachnoid barrier layer and a dura border cell layer"(4). The occurrence of traumatic SDG comprises $5-20 \%$ of space occupying posttraumatic lesions $(3,5)$. Based on clinical characteristics and serial CT scan finding, traumatic subdural hygroma (TSH) is divided into 4 types:

1.Resolution: Hygroma becomes smaller and smaller or the clinical signs gradually disappear (6).

2.Steadiness: Size of hygroma remains unchanged and 
no significant clinical changes occur after four weeks following head injury (6).

Improvement of patients with SDG types 1 and 2 by medical management is satisfactory and usually no surgical intervention is required (7).

3. Development: TSH, effusion and tissue density increases and the clinical signs gradually worsen (8).

4.Evolution:Hygroma evolves to chronic subdural hematoma $(\mathrm{CSDH})$ accompanied by increased intracranial pressure (6). Dura border cell layers can proliferate and form a neomembrane(9).Continuoussubdural effusion leads to bridge veins rupture and hygroma wall bleeding due to the gradual rise and capsule development.Also, fibrinolysis high performance results in impaired blood coagulation function, and thus CSDH is formed $(10,11)$.Transformation fromsubdural hygroma to chronic subdural hematomais well established (12). Surgery is taken into account as a modality in type 3 and 4 patients (6).

Subdural hygroma usually resolves spontaneously not needing surgical treatment $(8,13)$. Researchers have reported that more than $85 \%$ of SDG disappear within three months (3) but if the clinical condition deteriorates or subdural hygroma is associated with mass effect, it becomes a serious complication requiring surgical intervention $(14,15)$

\section{Methods and Materials/Patients}

This is an 11-year retrospective study in which records of 16 patients afflicted with traumatic subdural hygroma who were operated in Poursina Hospital in Rasht city (North of Iran) were studied. Patients' characteristics including age, sex, mechanism of injury and clinical symptoms were extracted from hospital records and analyzed. Initial examination of patients was performed through CT scan which was repeated during hospital stay. Information such as site and volume of hygroma, time of formation and presence of other concurrent skull damages were gathered and analyzed. Moreover, GCS on admission, at the time of hygromformation and at discharge were obtained in order to determine LOC. Clinical outcome was evaluated based on GOS at discharge. The patients were followed up using patient hospitalization records as well as interviews. The data were then analyzed using SPSS software (version 18) and Repeated Measures ANOVA. The differences were statistically significant at $\mathrm{P} \leq 0.05$.

\section{Results}

In this study, out of 16 included patients, 13 were men $(81.3 \%)$ and 3 women $(18.7 \%)$ ranging from 33 to 91 years of age (Mean age $=61,63 \pm 12.67 \mathrm{yrs}$ ). The etiology was car accident in half of the patients (50\%), 43.8\% fall and $6.3 \%$ assault. Head injury was severe in $18.8 \%$ of patients $(\mathrm{GCS}=3-8)$, moderate in $50 \%(\mathrm{GCS}=9-12)$ and mild in $31.3 \%(\mathrm{GCS}=13-15)$. A summary of information of SDG patients are presented in Tablel.The clinical signs and symptoms included fever $(56.3 \%)$, headache or dizziness $(25 \%)$, nausea and vomiting $(31.3 \%)$, convulsion $(56.3 \%)$, disturbance in consciousness $(62.5 \%)$ and mild hemiplegia hemiparesis(62.5\%). Each patient underwent at least three CT examinations when hospitalized. Subdural hygroma was diagnosed using the CT scans in which a crescent shape hypodense areawas observed. The average formation time of hygroma was $12.8 \pm 10.27$ days. In more than half of the patients, hygroma was formed on the sixth day or more. It was observed in $12.5 \%$ in fronto-temporal, $56.3 \%$ in frontoparietal and $18.8 \%$ in fronto-tempro-parietal of the subdural space. Hygroma was unilateral in $81.3 \%$ and bilateral in $18.8 \%$ of patients $(41.7 \%$ of patients right and $58.3 \%$ of patients left side) (Figure 1). The amount of the unilateral

Table 1. Information of SDG Patients

\begin{tabular}{|c|c|c|c|c|c|c|c|c|}
\hline No. & $\begin{array}{l}\text { Age } \\
\text { (yrs) }\end{array}$ & $\begin{array}{l}\text { Sex } \\
(\mathrm{F} / \mathrm{M})\end{array}$ & $\begin{array}{c}\text { GCS } \\
\text { Score On } \\
\text { Admission* }\end{array}$ & $\begin{array}{l}\text { GCS* } \\
\text { at Hygroma } \\
\text { Formation }\end{array}$ & $\begin{array}{c}\text { GCS* } \\
\text { at } \\
\text { Discharge }\end{array}$ & $\begin{array}{l}\text { Concomitant } \\
\text { Intracranial } \\
\text { Injuries*** }\end{array}$ & $\begin{array}{l}\text { Post-operative } \\
\text { Complications }\end{array}$ & $\begin{array}{c}\text { Patients } \\
\text { Outcome }(\mathrm{GOS}) * * *\end{array}$ \\
\hline 1 & 70 & Male & 12 & 10 & 15 & - & Hygroma recurrence & Moderate disability \\
\hline 2 & 61 & Male & 9 & 6 & 8 & SAH & - & Moderate disability \\
\hline 3 & 65 & Male & 14 & 13 & 14 & EDH & Hygroma recurrence & Moderate disability \\
\hline 4 & 91 & Male & 7 & 6 & 8 & DAI & - & Moderate disability \\
\hline 5 & 65 & Female & 10 & 8 & 14 & - & Cerebral edema & Moderate disability \\
\hline 6 & 54 & Male & 11 & 7 & 9 & Contusion & - & Moderate disability \\
\hline 7 & 60 & Female & 15 & 15 & 15 & - & Hygroma recurrence & Good recovery \\
\hline 8 & 55 & Male & 9 & 7 & 8 & DAI & - & Moderate disability \\
\hline 9 & 54 & Female & 14 & 13 & 15 & $\mathrm{EDH}$ & Hygroma recurrence & Good recovery \\
\hline 10 & 49 & Male & 8 & 6 & 7 & Contusion & - & Severe disability \\
\hline 11 & 66 & Male & 14 & 13 & 13 & Contusion & Cerebral edema & Moderate disability \\
\hline 12 & 72 & Male & 12 & 9 & 13 & SDH & - & Moderate disability \\
\hline 13 & 61 & Male & 9 & 7 & 7 & SAH & - & Moderate disability \\
\hline 14 & 56 & Male & 15 & 15 & 15 & SAH & Hydrocephaly & Good recovery \\
\hline 15 & 74 & Male & 12 & 11 & 13 & Contusion & Cerebral edema & Moderate disability \\
\hline 16 & 33 & Male & 7 & 6 & 9 & SDH & - & Moderate disability \\
\hline
\end{tabular}

*GCS: Glasgow Coma Scale

**SAH: Subarachnoid Hemorrhage, EDH: Epidural Hematoma, DAI: Diffuse Axonal Injury, Contusion: brain contusions, SDH: Subdural Hematoma

***GOS: Glasgow Outcome Scale 


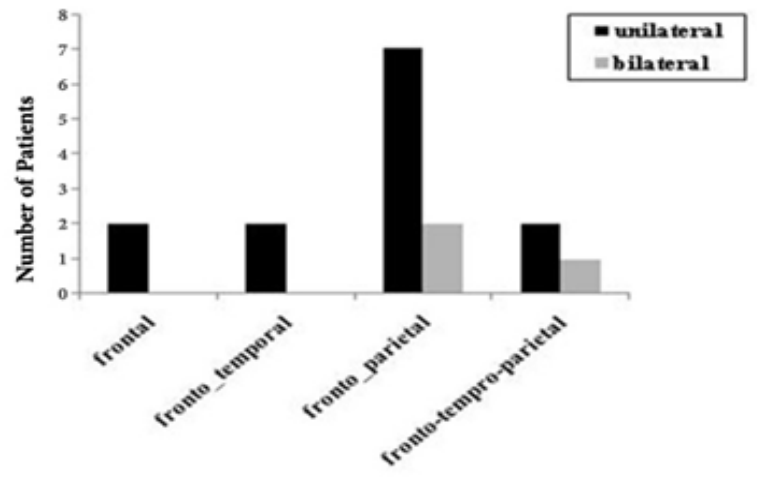

Figure 1. Location of Hygroma

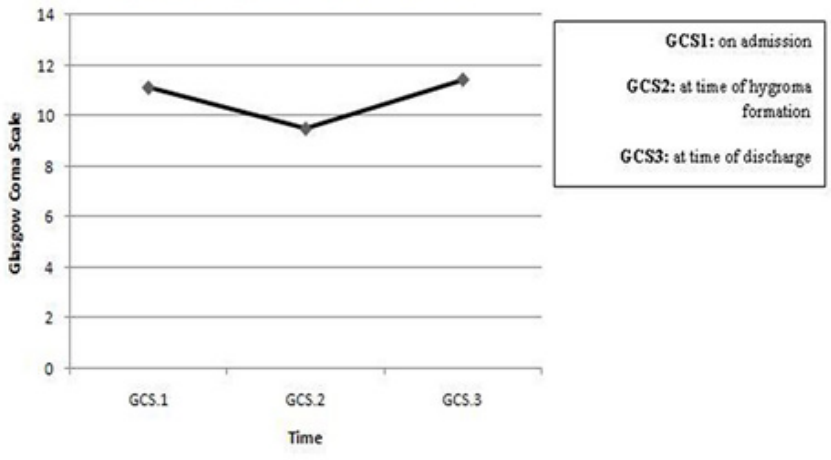

Figure 2. Changes in GCS at 3 Different Times hygroma was 30-42 $\mathrm{ml}($ Mean $=36.38 \pm 4.33 \mathrm{ml})$ and bilateral hygroma $13-55 \mathrm{ml}(\mathrm{Mean}=39.33 \pm 22.94 \mathrm{ml})$. The CT number of hygroma was 6-20 Hounsfield unit (Hu). Furthermore, most concomitant intracranial injuries in $\mathrm{CT}$ scans included cerebral contusion $(25 \%)$, subarachnoid hemorrhage (SAH) (18.8\%), epidural hematoma (EDH) (12.5\%), acute subdural hematoma (ASDH) (12.5\%) and diffuse axonal injury (DAI) $(12.5 \%)$. This study considered all patients who had undergone surgery due to subdural hyroma and those with medical treatment were excluded. In patients under general anesthesia, hygroma was extracted using a drain following burr hole Trephination procedure. GCS on admission ranged from 7 to 15 (Mean \pm SD; 11.12 \pm 2.78$)$, from 6 to 15 at hygroma formation (Mean $\pm \mathrm{SD} ; 9.5 \pm 3.37)$ and 7 to 15 at discharge (Mean $\pm \mathrm{SD} ; 11.44 \pm 3.24)$. The mean of GCS was statistically significant based on ANOVA in three times so that a significant difference was observed between the mean of GCS on admission time with discharge and time of hygroma formation $(\mathrm{P}<0.05)$ (Figure 2). All patients underwent subdural fluid drainage. Considering post-operative complications, $18.8 \%$ of patients had cerebral edema, $6.3 \%$ hydrocephaly and $25 \%$ had hygroma recurrence. Outcome was evaluated based on GOS after surgery at discharge time. The attributed scores were as follows: death (1), vegetative state (2), severe disability (3), moderate disability (4) andcomplete recovery (5). For statistical comparison, outcomes 1,2 and 3 were considered unfavorable and outcomes 4 and 5 as good outcomes. Therefore, $18.8 \%(\mathrm{n}=3)$ had good recovery (good outcome), $75 \%(\mathrm{n}=12)$ moderate disability, and $6.3 \%(\mathrm{n}=1)$ severe disability (unfavorable).

\section{Disscussion}

Subdural fluid accumulation will often disappear spontaneously and needs no surgical intervention (13). Based on prior research, sometimes these accumulations may cause intracranial pressure (ICP) or focal neurological deficit resulting in local compression on cerebral parenchyma (16). Though when subdural hygroma causes mass effect, surgical intervention becomes urgent (14), yet this is controversial. On the one hand, studies have shown that subdural accumulations are asymptomatic, insisting that only patients with worsening level of consciousness, increased intracranial pressure and headache could be diagnosed (15). Therefore, present work considered patients who underwent surgery due to subdural hygroma. Studies have revealed that subdural hygroma is mainly noted in old patients $(17,18,19,20)$. In our study alike, old patients were dominant (14 of 16 patients were over 50 years), because of expansion of subdural space secondary to cerebral atrophy (3). In our subjects, the mean interval time from head injury to hygroma formation was 12.8 days after head injury. In a study by Lee et al. (2000) subdural hygroma was diagnosed 11.6 days after head injury (9). Zanini et al. (2007) reported that the average time of hygroma formation was 9 days (12). Other similar findings were reported in other studies by this author (21). In our study in most patients, hygroma was formed on sixth day or after that. These finding suggest that hygroma is a delayed lesion. Many studies confirm our finding $(2,3,9,22)$. In the present study, CT findings showed that subdural hygroma was formed in fronto-parietal area in most patients. Also in all of our patients, most hygromas were formed in frontal area and to a lesser degree in other areas. Some prior studies also persist that subdural fluid accumulations occur in frontal area $(2,21)$. Lee et al. (1998) also believed that subdural hygromas usually place in frontalor temporal areas (3). In a study by Kim et al. (1979) it was seen that hygromas are mostly formed in frontal or fronto-temporal areas (23). Previous studies have indicated that this phenomenon can be due to the gravity of earth affecting brain since mostly all patients lie supine, causing the brain to gravitate occipitally (backward) which leads to hygroma formation in frontal area (3). Since the present study was conducted on patients who underwent surgery due to subdural hygroma, it is suggested that the leading mechanism resulting in hygroma formation is the cerebrocranial injury due to traumatic damages to the arachnoid layer by which CSF is directed into the subdural space gradually accumulated (6). Furthermore, studies have revealed that cerebrocranial injury can degrade the bloodbrain barrier and increase the permeability of blood capillary so that the plasma components are oozed and then accumulated gradually in the subdural space. As a result, the osmotic pressure is increased because of accumulation of high protein fluid in subdural space, absorbing water from the cerebral tissue and arachnoid space into the hygroma (6). By that, effusion and tissue density are increased and the clinical signs and symptoms become gradually worse and in most patients, surgical intervention becomes necessary (6). In our study, the decision for surgery was based mainly on LOC changes of patients, associated with growth of hygroma, which can occur through abovementioned mechanism. Along that, our results elucidated that LOC according to GCS is decreased at the time of hygroma formation compared to admission. Post-operative consciousness according to GCS showed that the LOC of patients increased compared to time of hygroma formation. These results explain the necessity and role of surgical treatment in improvement of 
LOC. One of the limitation of present study is its small sample size. Yet, the findings of this study can be considered as results of a pilot study. Thus, it is suggested future studies include a higher sample size.

\section{Conclusion}

This study showed that subdural hygroma is a delayed lesion that occurs after cerebro-cranial injury and in most patients resolves spontaneously without need for surgery. Yet, in certain cases, subdural fluid accumulations may gradually rise and the resulting mass effect on brain tissue would reduce LOC where drainage of hygroma is necessary and elevates consciousness. Therefore, the results of present study, though not comprehensive, tend to recommend surgery for patients with LOC due to hygroma and encourage future studies to consider a larger sample size.

\section{Acknowledgment}

The authors offer their special thanks to the Clinical Research Development Unit of Poursina Hospital, Guilan University of Medical Sciences, Rasht, Guilan, Iran.

\section{Funding}

None.

\section{Conflicts of Interest}

The authors declare that they have no conflicts of interest.

\section{References}

1. Salehpoor F, Meshkini A, Shokouhi Gh, et al. Prognostic Serum Factors in Traumatic Brain Injury: A Systematic Review. IrJNS. 2015;1(1):10-22.

2. Herold TJ, Taylor S, Abbrescia K, et al. Post-traumaticsubduralhygroma: case report. J Emerg Med. 2004; 27(4):361-366.

3. KS Lee. The pathogenesis and clinical significance of traumatic subdural hygroma. Brain Inj. 1998; 12(7) : 595-603.

4. Jeon SW, Choi JH, Jang TW, et al. Risk factors associated with subdural hygroma after decompressivecraniectomy in patients with traumatic brain injury: A comparative study. J Korean neurosurg Soc. 2011;49(6):335-338.

5. Sohn IT, Lee KS, Doh JW, et al. A Prospective Study on the Incidence, Patterns and Premorbid Conditions of Traumatic Subdural Hygroma. J Korean Neurosurg Soc. 1997; 26(1):87-93

6. Liu Y, Gong J, Li F, et al. Traumatic subdural hydroma: clinical characteristics and classification. Injury. 2009; 40(9):968-972.

7. Liu YG, Jia T, Liu M, et al. Type and its clinical characteristics of traumatic subdural hydroma. ZhonghuaWaiKeZaZhi. 2003; 41(10):763-765.

8. French BN, Cobb CA 3rd, Corkill G, et al. Delayed evolution of posttraumatic subdural hygroma. Surg Neurol. 1978; 9(3):145-148.

9. Lee KS, Bae WK, Bae HG, et al. The fate of traumatic subdural hygroma in serial computed tomographic scans. J Korean Med Sci.2000;15(5):560 568.

10. Feng JF, Jiang JY, Bao YH, et a. Traumatic subdural effusion evolves into chronic subdural hematoma: Two stages of the same inflammatory reaction?. Med Hypotheses. 2008;7(6):1147-1149.

11. Park SH, Lee SH, Park J, et al. Chronic subdural hematoma preceded by traumatic subdural hygroma. J ClinNeurosci. 2008;15(8):868-872.

12. Zanini MA, Resende LA, Freitas CC, et al. Traumatic subdural hygroma: five cases with changed density and spontaneous resolution. ArqNeuropsiquiatr. 2007;65(1):68-72.

13. Kentaro Mori, Minoru Maeda. Delayed magnetic resonance imaging with Gd-DTPA differentiates subdural hygroma and subdural effusion. Surgical Neurology. 2000;53(4):303-311.

14. Paiva WS, Oliveira AM, de Andrade AF, et al. Remotepostoperativeepiduralhematomaaftersubduralhygromadrainage. Case Rep Med. 2010; doi: 10.1155/2010/417895.

15. Wang HK, Lu K, Liang CL, et al. Contralateralsubduraleffusionrelated to decompressivecraniectomyperformed in patients with severetraumatic brain injury. Injury. 2012; 43(5):594-597.
16. Caldarelli M, Di Rocco C, Romani R. Surgical treatment of chronicsubduralhygromas in infants and children. ActaNeurochir (Wien). 2002; 144(6):581-588

17. Coșar M, Eser O, Aslan A, et al. Rapid resolution of acute subdural hematoma and effects on the size of existent subdural hygroma: a case report. Turk Neurosurg. 2007 ;17(3):224-227.

18. Danil A. Posttraumatic extracerebral fluid collections. Romanian Neurosurgery. 2013; 20(2):139-148.

19. Ishibashi A, Yokokura Y, Miyagi J. Clinical analysis of nineteen patients with traumatic subdural hygromas. Kurume Med J. 1994; 41(2):81-85.

20. Tsuang FY, Huang AP, Tsai YH, et al. Treatment of patients with traumatic subdural effusion and concomitant hydrocephalus. J Neurosurg. $2012 ; 116(3): 558-565$.

21. Zanini MA, de Lima Resende LA, de Souza Faleiros AT, etal.Traumatic subdural hygromas: proposed pathogenesis based classification. J Trauma.2008;64(3):705-713.

22. Lang JK, Ludwig HC, Mursch K, et al. Elevated cerebral perfusion pressure and low colloid osmotic pressure as a risk factor for subdural space-occupying hygromas?. Surg Neurol. 1999; 52(6):630-637.

23. Kim SJ, Rhee BA, Lee YK. Clinical evaluation of the traumatic subdural hygroma. J Korean Neurosurg Soc. 1979; 8(2):255-260.

\section{Comments}

Yousefzadeh-Chabok and colleagues operated 16 patients with traumatic subdural hygroma (TSH) (1). Fifteen patients had good outcome and one had poor outcome. There were 4 out of $16 \mathrm{TSH}$ recurrences. Authors mentioned that $85 \%$ of the TSH patients resolve within three months (2). To determine the effectiveness of surgery on TSH, we need a control group of TSH patients who managed without surgery. Otherwise, we cannot claim the effectiveness of surgery on TSH. Figure 2 shows small decrease in Glasgow Coma Scale (GCS) following TSH formation. There is no statistical test to show if the small decrease in GCS was significant. In addition, there is no high quality evidence to show in each case if the TSH is progressive or may be resolved spontaneously.

Vafa Rahimi-Movaghar, MD, Professor of Neurosurgery, Sina Trauma and Surgery Research Center, Tehran University of Medical Sciences, Tehran, Iran

\section{References}

1. Yousefzadeh-Chabok S, Hosseinpour M, Mohtasham-Amiri Z, Alijani B. The Role of Surgical Treatment in Traumatic Subdural Hygroma: A Pilot Study. IrJNS. 2015;1(2): 40-43

2. Lee KS. The pathogenesis and clinical significance of traumatic subdural hygroma. Brain Inj. 1998; 12(7) : 595-603. 